\title{
F-18 Fluciclovine Positron Emission Tomography and Computed Tomography
}

National Cancer Institute

\section{Source}

National Cancer Institute. F-18 Fluciclovine Positron Emission Tomography and

Computed Tomography. NCI Thesaurus. Code C148230.

An imaging procedure that combines fluciclovine F18 PET with an computed tomography to overlay activity data onto detailed anatomic images. 